\title{
Chemovirotherapy: combining chemotherapeutic treatment with oncolytic virotherapy
}

This article was published in the following Dove Press journal:

Oncolytic Virotherapy

23 February 2015

Number of times this article has been viewed

\author{
Eike Binz \\ Ulrich M Lauer \\ Department of Internal \\ Medicine, Division of Hepatology, \\ Gastroenterology, Infectiology, \\ Medical University Hospital, \\ Tuebingen, Germany
}

Correspondence: Ulrich M Lauer University Hospital Tuebingen, Department of Internal Medicine, Division of Hepatology,

Gastroenterology, Infectiology,

Otfried-Mueller-Str I0, D-72076

Tuebingen, Germany

Tel +49 707I 2983190

Fax+49 707I 294686

Email ulrich.lauer@uni-tuebingen.de

\begin{abstract}
Oncolytic virotherapy has made significant progress in recent years, however, widespread approval of virotherapeutics is still limited. Primarily, this is due to the fact that currently available virotherapeutics are mostly tested in monotherapeutic clinical trials exclusively (ie, not in combination with other therapies) and so far have achieved only small and often clinically insignificant responses. Given that the predominantly immunotherapeutic mechanism of virotherapeutics is somewhat time-dependent and rapidly growing tumors therefore exhibit only minor chances of being captured in time, scenarios with combination partners are postulated to be more effective. Combinatory settings would help to achieve a rapid stabilization or even reduction of onset tumor masses while providing enough time (numerous months) for achieving immuno(viro)therapeutic success. For this reason, combination strategies of virotherapy with highly genotoxic regimens, such as chemotherapy, are of major interest. A number of clinical trials bringing the concepts of chemotherapy and virotherapy together have previously been undertaken, but optimal scheduling of chemovirotherapy (maximizing the anti-tumor effect while minimizing the risk of overlapping toxicity) still constitutes a major challenge. Therefore, an overview of published as well as ongoing Phase I-III trials should improve our understanding of current challenges and future developments in this field.
\end{abstract}

Keywords: cancer, combination therapy, chemotherapy, virotherapy, oncolytic virus, clinical trials

\section{Introduction}

Currently, wild-type as well as recombinant oncolytic viruses (OVs) are intensely investigated as novel biological agents for the treatment of cancer. The basic principle of this virotherapeutic treatment modality is the selective infection of cancerous tissues while leaving healthy tissues unharmed. Within the infected tumor cells, virotherapeutics are now able to potently replicate, which ultimately leads to massive tumor cell lysis (oncolysis), and an intense danger signal and stimulus to the immune system by a concomitant release of both tumoral as well as viral antigens. Subsequently, newly released progeny virotherapeutics are capable of targeting uninfected neighboring or distant tumor cells which results in an infinite number of oncolytic cycles and the eradication of all tumorous tissues. If this process of direct and rapid tumor eradication (within weeks) on its own is not efficient enough, it is anticipated that stimulation of the immune system would lead to a long-lasting anti-tumoral immune response within a number of months.

Despite its promising potential in targeted anti-cancer therapy not one monotherapeutic application of OVs (applied without combination partners) has been approved 
in the western hemisphere so far. This is due to the fact that current oncolytic monotherapies have demonstrated success in single patients, ${ }^{1}$ but have failed to show any significant clinical benefit for the majority of virotherapeutic treated cancer patients. This is not unsurprising, as single agent treatments of cancers have rarely been found to result in a deceleration of tumor progression or even lasting reduction of tumor masses. This clearly indicates the existence of primary as well as secondary adapted resistance to the large array of virotherapeutic vector families currently being clinically evaluated. In this context, tumor cell subpopulations evolve in the course of monotherapeutic regimens (such as oncolytic virotherapy), armed with distinct genetic alterations that render them not only i) resistant to common virotherapeutic agents, but also ii) cross-resistant to other virotherapeutic compounds and furthermore, iii) concealing them against any initially induced anti-cancer immune response.

In contrast, most preclinical data suggest that virotherapy can be ideally combined with other treatment options, such as chemotherapy. ${ }^{2}$ Combinatorial treatment regimens exhibit diverse mechanisms of action and thereby more often constitute a successful approach to overcome such resistance mechanisms. Beyond that, potential synergistic interactions between the combined agents permit possible dose reductions of the applied agents, both to a less toxic as well as, and importantly, to a less costly degree. Current clinical data suggest that combining OVs with already approved chemotherapeutic treatment modalities might be an important key in unlocking the true potential of virotherapeutics while preventing the induction of resistance mechanisms. Since the mechanism of action of OVs is believed to be mostly immunotherapeutic in nature, two prerequisites are postulated for success of this novel biological cancer therapy approach: i) when starting virotherapy, overall tumor masses should be low (since immunotherapy can take months to be effective and more likely works in patients with stabilized tumor diseases) and ii) there is an urgent necessity for balancing/ curbing especially rapidly growing tumors, preferably already prior to the onset of the virotherapeutic treatment. Both prerequisites often can be achieved by modern chemotherapies, particularly in first line scenarios.

This review primarily focusses on the rare clinical studies in which the concepts of chemotherapy and virotherapy have to date been combined (Figure 1), leading to an early classification of chemovirotherapeutic combination regimens. In this approach, we specifically paid attention to chemovirotherapeutic combinations employing standardof-care chemotherapeutics, tyrosine kinase inhibitors and monoclonal antibodies, while excluding non-standard regimens (comprising prodrug-activating enzymes, vaccinedriven approaches, and epigenetic agents or other small molecules).

\section{Current state of clinical chemovirotherapeutic trials}

To date, only a small number of clinical trials involving the combination of standard chemotherapy with virotherapeutic agents (chemovirotherapeutic regimens), have been completed and published; the majority are either finished and not yet published, ongoing, or still in their recruitment or

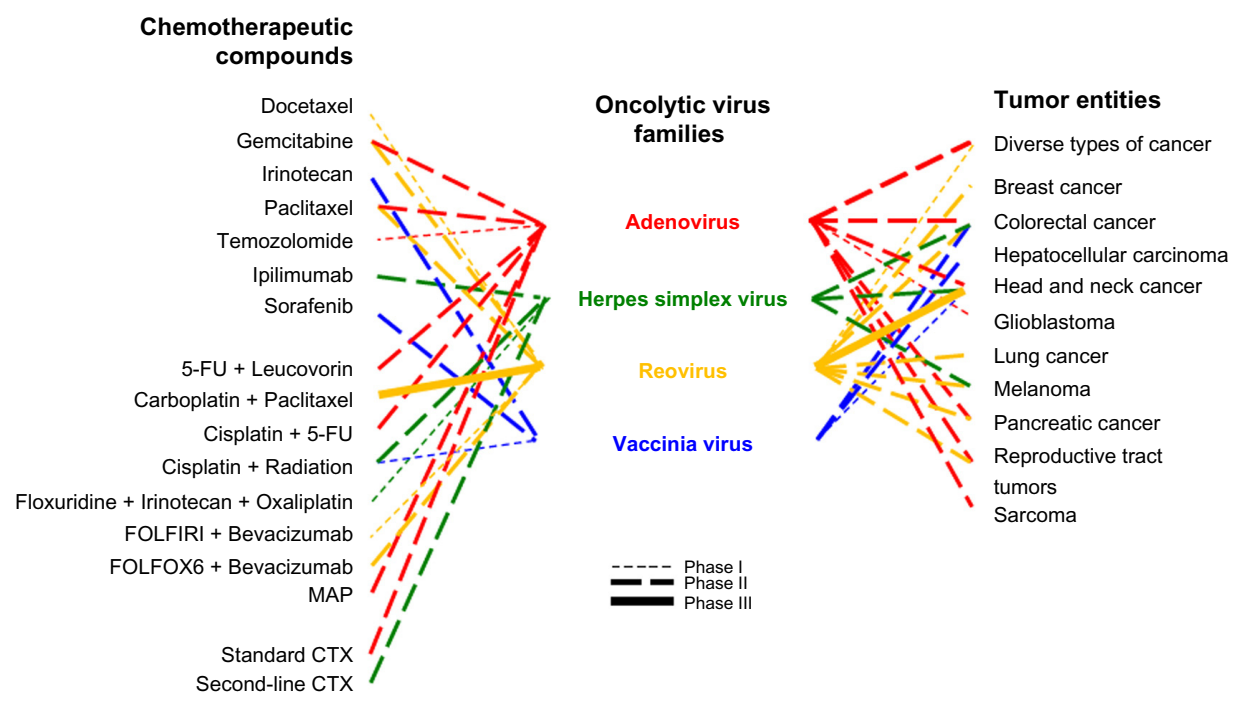

Figure I Chemovirotherapy combination regimens currently investigated in clinical trials.

Abbreviations: 5-FU, 5-fluorouracil; CTX, chemotherapy; FOLFIRI, folinic acid (leucovorin) + 5-fluorouracil + irinotecan; FOLFOX6, folinic acid (leucovorin) + 5-fluorouracil + oxaliplatin; MAP, mitomycin C + adriamycin (doxorubicin) + cisplatin. 
planning phases (Tables 1-3). Some virotherapeutic agents used in these chemovirotherapeutic trials have therefore reached a more advanced stage, in both translational development and clinical testing, which can be mainly attributed to the onset of vector construction as well as the respective history of preclinical development. Since monovirotherapeutic approaches employing vectors of adeno- and herpesvirus origin are by far the ones most extensively used in clinical practice, they also represent the largest fraction in our array of already completed and published clinical trials performed in this specific combinatorial (ie, chemovirotherapeutic) setting. Due to the encouraging data from a Phase II study with reovirus applied in head and neck cancer patients, ${ }^{3}$ the chemovirotherapeutic combination of reovirus with carboplatin and paclitaxel is currently the only one which has reached the status of a Phase III trial (REO 018). This study has recently been completed and shows some interesting results, albeit final data for overall survival (OS) are still awaited. ${ }^{4}$

\section{Virus families evaluated in early clinical trials (Phase I studies)}

An overview on completed and currently ongoing chemovirotherapeutic Phase I trials is given in Table 1 sorted by corresponding virus families. These Phase I trials primarily aim at obtaining knowledge on vector safety profiles as well as optimized dosing regimens. While the preclinical evidence seen in tumor cell culture, immunodeficient and more importantly immunocompetent animal tumor models strongly suggests the benefit of combinatorial chemovirotherapeutic regimens, only a small number of these preclinically defined schedules have made it to the bedside so far. In fact, each vector/virus family and moreover each single chemovirotherapeutic Phase I protocol faces its own/specific difficulties collecting sufficient clinical evidence to move forward to Phase II.

\section{Adenovirus-based chemovirotherapeutic regimens}

Combination protocols incorporating adenovirus-based virotherapeutics (adenovirotherapeutics) were the first to enter the clinical stage and therefore faced the pioneering task of responding to concerns about safety and tolerability of this combinatorial approach, as well as to demonstrate the clinical benefits of chemoadenovirotherapeutic regimens. Currently the most prominent vector system (and also the first virus applied in a combinatorial chemoadenovirotherapeutic setting) is ONYX-015; an adenovirus exhibiting a deletion in the E1B 55-kDa gene. ${ }^{5}$ Phase I trials of ONYX-015 in patients with gastrointestinal tumors metastatic to the liver, ${ }^{6}$ unresectable pancreatic carcinoma, ${ }^{7}$ and advanced sarcomas ${ }^{8}$ were the first to show positive results from this approach and exhibited no enhanced toxicity profiles from combinatorial treatments. These approaches have therefore rapidly moved forward to Phase II trials ${ }^{9,10}$ and now put the primary focus on the read-out of anti-tumoral efficiencies.

Since all of these early adenovirotherapeutic Phase I/II trials applied ONYX-015 either locally or regionally (not systemically) to tumor tissues (via intratumoral injection or by intra-arterial injection into the hepatic artery), one of the next tasks would have been to prove the safety of systemic application regimens to more efficiently affect and eliminate distal metastases and, broaden the spectrum of treatable tumor entities. However, when a Phase III trial incorporating ONYX-015 was dropped in 2003, in part to devote its resources to a more conventional drug which then was awaiting approval from the US Food and Drug Administration (FDA), further clinical development on this particular agent was stopped.

In a next step of further development of adenovirus-based chemovirotherapeutic regimens the intratumoral application of H101, a vector being quite similar to ONYX-015, ${ }^{11}$ received marketing approval in the People's Republic of China in 2005. ${ }^{12}$ Notably, Phase II/III ${ }^{13,14}$ trials demonstrated an enhanced anti-tumor effect of $\mathrm{H} 101$ in combination with cisplatin and 5-fluorouracil (5-FU) compared to standard chemotherapy with cisplatin and 5-FU alone, for the treatment of patients with head and neck cancer. However, the results for survival and clinical benefit were not adequately collected. ${ }^{12}$ To date H101 still represents the only approved viral agent globally, but is currently only in use in the People's Republic of China. No other chemovirotherapeutic regimens incorporating novel adenoviral vector systems have found their way into the clinic.

Only recently, combinations with refined adenoviral vectors such as ColoAd1, DNX1401, and VCN-01 entered the stage of early clinical development (Tables 1 and 2). They are now being assessed for their safety and more importantly their clinical benefit compared to current monotherapeutic virotherapies, as well as their improved clinical outcomes and potential to supersede previously investigated combinatorial treatment regimens.

\section{Herpes simplex virus (HSV)-based chemovirotherapeutic regimens}

Numerous preclinical studies have been performed combining different vector constructs of the HSV virus family with 


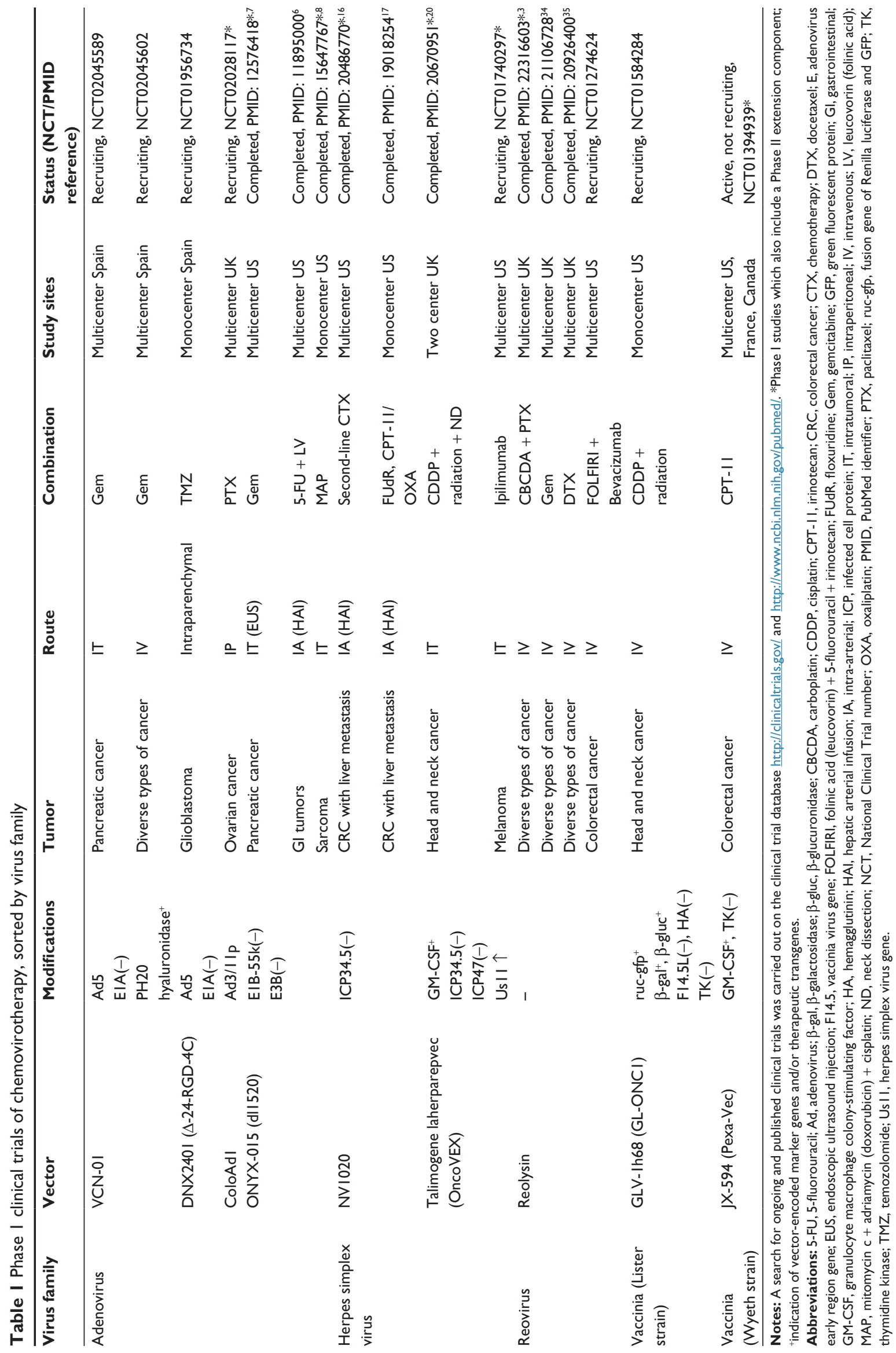




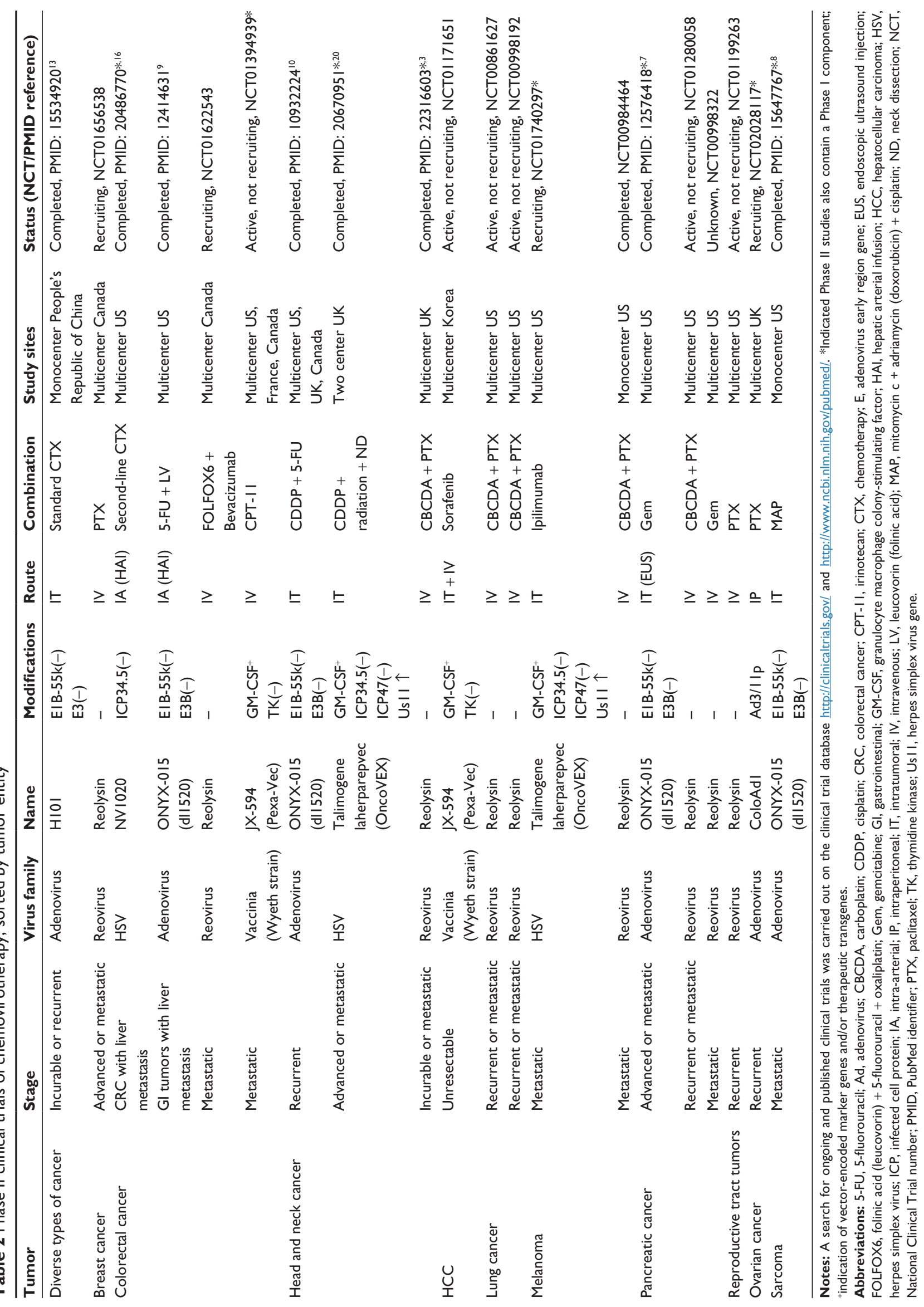


Table 3 Current Phase III clinical trials of chemovirotherapy

\begin{tabular}{|c|c|c|c|c|c|c|c|}
\hline Name & Tumor & Patients & Combination & Route & Sequence & Study sites & $\begin{array}{l}\text { Status (NCTI } \\
\text { PMID reference) }\end{array}$ \\
\hline $\begin{array}{l}\text { REO } 018 \\
\text { (Reolysin) }\end{array}$ & $\begin{array}{l}\text { Head and } \\
\text { neck cancer }\end{array}$ & $\begin{array}{l}\text { Recurrent/metastatic } \\
\text { platinum refractory } \\
\text { squamous cell carcinoma } \\
\text { Patient subgroup I: } \\
\text { locoregional tumors } \\
\text { with or without } \\
\text { metastases ( } n=1 \text { I8) } \\
\text { Patient subgroup 2: } \\
\text { only measured distal } \\
\text { metastases ( } n=47 \text { ) }\end{array}$ & $\mathrm{CBCDA}+\mathrm{PTX}$ & IV & $\begin{array}{l}\text { Day I: PTX } 3 \text { h, } \\
\text { CBCDA } 0.5 \text { h } \\
\text { Days I-5: } \\
\text { Reolysin daily I h } \\
\text { (2I days per cycle) } \\
\text { Control arm: } \\
\text { CTX + IV placebo } \\
\text { (no virus) }\end{array}$ & $\begin{array}{l}\text { Multicenter US, Russia, } \\
\text { UK, Spain, Hungary, } \\
\text { Canada, Italy, Poland, } \\
\text { Belgium, France, Germany, } \\
\text { Greece, Slovenia }\end{array}$ & $\begin{array}{l}\text { Completed, } \\
\text { NCTOII } 66542\end{array}$ \\
\hline
\end{tabular}

Notes: A search for ongoing and published clinical trials was carried out on the clinical trial database http://clinicaltrials.gov/ and http://www.ncbi.nlm.nih.gov/pubmed/. Further data on the study design of the REO 018 trial are from Oncolytics Biotech (http://www.oncolyticsbiotech.com/English/clinical-trials/clinical-trials-news/clinical-trialsdetails/20I3/REO-0I8/).

Abbreviations: CBCDA, carboplatin; CTX, chemotherapy; IV, intravenous; PTX, paclitaxel; h, hour(s); NCT, National Clinical Trial number; PMID, PubMed identifier.

a plethora of different chemotherapeutic agents (recently reviewed by Kanai et al). ${ }^{15}$ The evidence of synergistic interactions seen in many preclinical chemovirotherapeutic protocols seems to be overwhelming. However, only a few of these preclinical regimens have so far reached the stage of clinical evaluation.

One of the viral agents currently under clinical investigation in Phase I/II trials is NV1020 which is infused directly into the hepatic artery prior to second-line chemotherapies in patients exhibiting advanced liver-dominant metastatic colorectal cancer (mCRC). ${ }^{16,17}$ In these trials, in which the chemotherapeutic agents were given only after NV1020 was applied via hepatic artery infusion (either as a singleshot ${ }^{17}$ or by four weekly doses), ${ }^{16}$ additional benefits of the chemovirotherapeutic combination were observed leading to the conclusion that NV1020 stabilizes liver metastases in mCRC with minimal toxicity. Carcinoembryonic antigen (CEA) levels, correlating with tumor regression, dropped further ${ }^{17}$ and the overall clinical control rate could be pushed from initially $50 \%$ to $68 \%$ after second-line chemotherapy. ${ }^{16}$

Another HSV-based virotherapeutic is talimogene laherparepvec ${ }^{18}$ (T-VEC, OncoVEX), which represents one of the most clinically advanced approaches of mono(viro)therapy. Results from a completed Phase III trial treating advanced stages of melanoma were able to confirm the expectations. The T-VEC virotherapeutic is currently undergoing FDA and European Medicines Agency approval for monovirotherapy. ${ }^{19}$ Interestingly, the chemovirotherapeutic combination of neoadjuvant T-VEC with cisplatin, radiation and ultimately radical neck dissection (6-10 weeks after the chemovirotherapeutic protocol) in patients with head and neck cancer ${ }^{20}$ initially resulted in a radiologic response rate of $82 \%$, while the pathologic complete response rate after neck dissection was $93 \%$. Therefore, T-VEC is expected to be developed further for chemovirotherapeutic application regimens.

A Phase Ib/II trial in melanoma patients combining T-VEC with the checkpoint blockade inhibitor ipilimumab is currently ongoing. Preliminary Phase Ib results, published at the 2014 American Society of Clinical Oncology annual meeting, ${ }^{21}$ indicate higher response rates for this immunovirotherapeutic approach than with either agent alone. Therefore, the results of the ongoing Phase II part of this combinatorial study are eagerly awaited. Future chemovirotherapeutic regimens with ipilimumab as a further add-on are under development.

\section{Reovirus-based chemovirotherapeutic regimens}

Reolysin (recently reviewed by Clements et al) ${ }^{22}$ contains a naturally occurring, ubiquitous, non-enveloped human reovirus strain which replicates in KRAS-mutant cells resulting in profound tumor cell lysis. ${ }^{23}$ In a recent Phase I dose escalation study, a standard FOLFIRI (folinic acid (leucovorin) +5 -fluorouracil + irinotecan) chemotherapy regimen was administered parallel with escalating Reolysin doses in patients with KRAS-mutant mCRC. ${ }^{24}$ As a result, the chemovirotherapeutic combination of FOLFIRI and Reolysin was found to be safe and resulted in disease control in the majority of patients, including those who previously progressed on irinotecan. ${ }^{24}$ Due to these encouraging results, additional studies are planned. 


\section{Vaccinia virus-based chemoviro- therapeutic regimens}

Due to its role in the worldwide smallpox eradication program Vaccinia virus represents the vaccine virus with the longest clinical use and therefore the largest clinical experience. In cancer treatment the clinically most advanced members of this virus family (recently reviewed by Guse et $\mathrm{al}^{25}$ are JX-594 (Pexa-Vec), encoding for the immunomodulatory cytokine GM-CSF, ${ }^{26}$ and GL-ONC1 (also known as GLV-1h68), encoding for multiple marker genes. ${ }^{27}$ The combination of JX-594 with the oral multiple kinase inhibitor sorafenib in patients with hepatocellular carcinoma was investigated first in a pre-Phase I evaluation, ${ }^{28}$ later on in a Phase II setting finally leading to the current design of a Phase III trial in which Pexa-Vec application will be followed by sorafenib in the first-line treatment of patients with advanced hepatocellular carcinoma. ${ }^{29}$ This study is expected to start recruitment by mid-2015.

Another Phase I trial currently investigates GL-ONC1 in combination with cisplatin and radiation in patients with locoregional advanced head and neck cancer. According to a recent publication of first data, delivery of GL-ONC1 was found to be safe and feasible in 12 patients with advanced head/neck cancer undergoing standard chemoradiotherapy plus virotherapy. ${ }^{30}$ However, further studies are needed to determine the optimal dosing schedule for future Phase II testing.

\section{Chemovirotherapeutic regimens tested in Phase II studies}

Table 2 represents an overview of the tumor entities which so far have been addressed by combinatorial chemovirotherapeutic treatment regimens in more advanced (ie, Phase II) settings.

Most of these chemovirotherapeutic Phase II trials encompass tumor entities with high medial need (such as malignant melanoma, pancreatic cancer, or ovarian cancer with peritoneal carcinomatosis), that are associated with unsatisfactory current standard of care therapies and result in poor survival rates and severe side effects due to the use of highly genotoxic compounds. Other chemovirotherapeutic Phase II trials focus on tumor entities that affect a high number of patients ("high volume" cancer diseases, such as breast, colorectal, and lung cancer) which are recognized "traditionally" as major targets of experimental cancer drug development.

While most of the completed Phase II trials displayed in Table 2 utilized combinations of the adenoviral vectors
ONYX-015 or H101 with different chemotherapeutic agents ( $n=5 / 9$; recently reviewed by Chen et al), ${ }^{31}$ two thirds of currently ongoing Phase II trials represent combination regimens employing reovirus $(n=7 / 11)$. The combination protocol of carboplatin and paclitaxel $(n=3 / 7)$ used in these chemovirotherapeutic trials was derived from data from an earlier Phase I/II trial in which diverse types of cancer (Phase I) and later patients exhibiting a single tumor type (head and neck cancer; Phase II) were treated. ${ }^{3}$ The results from the Phase II trials resulted in the implementation of a chemovirotherapeutic Phase III trial (REO 018), and Phase II trials addressing melanoma, pancreatic, and lung cancer patients were initiated in the hope of treating other tumor entities equally if not more successfully. Chemovirotherapeutic combination protocols encompassing other well-known vector systems such as the adenovirotherapeutic ColoAd1, the herpesvirotherapeutic T-VEC, and the poxvirotherapeutic JX-594 are currently in Phase II testing.

Since T-VEC i) was applied intratumorally to metastatic melanoma patients, ii) expresses the immunostimulatory molecule GM-CSF, and iii) melanoma is known to be highly susceptible to immunotherapy, the success of this treatment protocol in metastatic patients likely depends on the activation and amplification of anti-cancer immunity.

\section{Chemovirotherapeutic regimens currently tested in Phase III studies}

While most of the chemovirotherapeutic combination regimens currently under investigation represent Phase I/II trials, only a single chemovirotherapeutic setting has recently reached Phase III status (Table 3 ). This trial, designated as REO 018, ${ }^{32}$ was designed as a two-stage adaptive Phase III study combining carboplatin and paclitaxel with intravenous administration of reovirus (versus carboplatin, paclitaxel, and placebo in the control arm) in patients with second-line, platinum-refractory, taxane-naïve head and neck cancer. This study was found to be safe and well-tolerated. ${ }^{32}$ Recently, an intent-to-treat analysis of the OS of the 118 patients with locoregional disease was performed on all patients to the median progression free survival in each arm, in which 32 patients receiving post-discontinuation therapy with other agents were censored at the date on which they commenced the first of these post-discontinuation therapies. ${ }^{4}$ This analysis demonstrated a statistically significant improvement in OS for the test arm versus the control arm. When looking at the patients with distal metastases alone ( $n=47$ patients), there were too few patients at the time of the analysis to power a statistical analysis of the progression free survival and OS. 
Final data for the entire REO 018 study are awaited. Based on this data, a randomized, follow-on Phase III registration study in patients with recurrent head and neck cancers is planned, which will take the place of the planned second stage of the originally scheduled REO 018 clinical trial. ${ }^{4}$

\section{Conclusions and future directions}

Current clinical trials on chemovirotherapy are based on a plethora of encouraging results from numerous preclinical models and are obtained mostly by systematic approaches in which key features such as the order of application (virus first versus chemo first versus concomitant use of virus/ chemo) have been varied and optimized. However, to directly extrapolate clinical benefit from murine data has proven to be diffcult since experimental tumors often grow to a size requiring euthanasia within a few weeks and therefore experiments generally run on a tight timeline. Chemotherapy is given as single or multiple doses, with schedules that barely resemble what is used in the clinic. Furthermore, human tumor xenografts cannot easily be used in immunocompetent mice, which limits the utility of murine testing of clinically relevant chemovirotherapy combinations. Therefore, translating the preclinical knowledge to positive, practice-changing clinical trials constitutes a great challenge.

The approach of current clinical trials, to investigate a large array of tumor entities with different chemovirotherapeutic regimens, could be regarded as somewhat unsystematic in nature. As such, important details of the respective chemovirotherapeutic application settings have, to date, not been standardized. Standardization of scheduling seems to be important in order to maximize the antitumoral effects while minimizing overlapping toxicity/ adverse effects. These considerations are also relevant for the standardization of application methods employed in the use of virotherapeutics (ie, directly [intratumorally] or systemically [intravenously]), for use in this specific combinatorial setting of chemovirotherapy. It has to be conceded that endless combinations and varying schedules would be too time consuming and expensive to test in the clinical setting. The challenge is, therefore, to find a balance between the isolated development of clinical chemovirotherapeutic regimens and the systematic testing, monitoring, and optimization of different schedules.

The concerns expressed earlier regarding safety and overlapping toxicity profiles of combined chemotherapeutic and virotherapeutic treatment (especially when given concomitantly) have dampened. Naturally each single combination protocol has to undergo preliminary testing in a clinical
Phase I setting in which the main focus is therapy-related adverse effects, but present data from the multiplicity of tested chemovirotherapeutic protocols have been reassuring. Virotherapeutic treatment typically leads to flu-like symptoms (including fever, myalgia, asthenia and/or chills) shortly after virus injection, but the grade and duration of these symptoms remained mostly low even when patients were treated with concurrent chemotherapy. Levels of leukopenia and mucositis, adverse effects seen typically after chemotherapeutic treatment, were not significantly altered. In fact, it remains difficult to fully distinguish between chemotherapy and virotherapy induced toxicity. The fact that the mechanism of action between these two therapeutic principles differ greatly might explain why the recorded adverse effects were not significantly aggravated by the combination compared to either agent alone.

The mechanisms underlying the anti-tumoral effects of the most common virotherapeutic agents and their combination with chemotherapeutics still remain mostly unknown. Many of the variables associated with the effective combination of these two distinct modes of action might be elucidated with the right scheduling. Important questions, such as potential implications of chemotherapeutics on i) viral replication and/or ii) the immunotherapeutic effects potentially triggered by virotherapeutics, have not yet been addressed. The oncolytic paradigm, in which virus-mediated oncolysis of tumor cells results in the release of a broad spectrum of immunostimulatory molecules such as viral and tumor antigens as well as markers of aberrant cell death, clearly states the requirement of such an anti-tumor immune response to achieve a sustained therapeutic effect. While a potent anti-immune response is desperately needed in the long-term, too strong an initial response after antiviral infection might affect the capability of OVs to infect and replicate in tumor cells, constituting the premise for oncolysis and the release of cellular danger signals. Chemotherapeutics, besides their direct anti-tumor effects, are known to affect the immune system in many ways and in the context of finding an effective combination with immuno(viro)therapeutics, the importance of extending our focus to these mechanisms becomes more obvious. ${ }^{33}$

Before embarking on large Phase III studies, Phase I/II chemovirotherapy trials specifically investigating the complex balance of beneficial and detrimental effects on the immune response, and on the replication of virotherapeutics, are therefore of great interest. Conversely, given that immunotherapeutic approaches can take months to be effective they are more likely to work in patients with stabilized tumor diseases. Still it is not known to what extent 
tumor cell death is required and should be achieved by the genotoxic chemotherapeutics in order to pave the way for the immunovirotherapeutic component to take hold in these chemovirotherapeutic approaches. Thus, questions regarding the scheduling of both therapies should be addressed more extensively in future study designs.

Given that OVs are relatively new agents, with only limited clinical applications in recent years, trials investigating this treatment modality in monotherapeutic or combinatorial settings naturally include only patients at their end-stage of disease (after all other treatment modalities were exploited but unable to achieve the desired anti-tumor effect). This situation is clearly illustrated by the summation of combinatorial treatment protocols addressing the different tumor entities in a Phase II setting, as displayed in Table 2, but holds significance for all trials involving OVs. The immune system of endstage cancer patients however, may be considered to be less potent and might not be the best partner in aiding oncolytic virotherapy to unfold to its full potential. Since numerous clinical trials have so far demonstrated both safety as well as tolerability of viral agents in combination with chemotherapeutics, a next step of chemovirotherapeutic development would be to address earlier tumor stages as well (ie, in the setting of first-line chemovirotherapeutic regimens).

In this context, the read-out of any anti-tumoral efficacy in complex study settings, encompassing such diverse combination partners as genotoxic chemotherapeutic compounds and virotherapeutics, has to be considered as highly challenging. Numerous patients who have undergone a chemovirotherapeutic study treatment with subsequent tumor progression (measured clinically and/or with imaging modalities) will receive further therapies with other (mostly genotoxic) agents. This problem has been illustrated in the recent REO 018 study in which patients receiving post-discontinuation therapy with other agents were censored at the date on which they commenced the first of these post-discontinuation genotoxic therapies. Similar patterns of analysis in other chemovirotherapeutic studies might be required as well, in order to obtain conclusive study results.

All in all, combining chemotherapy and virotherapy in the clinical setting is not expected to be easy, but there is value in further optimizing the known treatment parameters and attempting to achieve major progress in this highly important field of advanced cancer therapy.

\section{Acknowledgments}

We are very grateful to Julia Beil, Susanne Berchtold, and Zoe Squire for critical reading of the manuscript.

\section{Disclosure}

The authors have no conflicts of interest to disclose.

\section{References}

1. Russell SJ, Federspiel MJ, Peng KW, et al. Remission of disseminated cancer after systemic oncolytic virotherapy. Mayo Clin Proc. 2014; 89(7):926-933.

2. Woller N, Gurlevik E, Ureche CI, Schumacher A, Kuhnel F. Oncolytic viruses as anticancer vaccines. Front Oncol. 2014;4:188.

3. Karapanagiotou EM, Roulstone V, Twigger K, et al. Phase I/II trial of carboplatin and paclitaxel chemotherapy in combination with intravenous oncolytic reovirus in patients with advanced malignancies. Clin Cancer Res. 2012;18(7):2080-2089.

4. Oncolytics Biotech. Oncolytics Biotech Inc. Announces Additional Data from REO 018 Randomized Study of REOLYSIN in Head and Neck Cancers [press release]. Calgary: Oncolytics Biotech; April 8, 2014. Available from: http://www.oncolyticsbiotech.com/ news/press-release-details/2014/Oncolytics-Biotech-Inc-AnnouncesAdditional-Data-from-REO-018-Randomized-Study-of-REOLYSINin-Head-and-Neck-Cancers. Accessed December 4, 2014.

5. Kirn D. Clinical research results with d11520 (Onyx-015), a replicationselective adenovirus for the treatment of cancer: what have we learned? Gene Ther. 2001;8(2):89-98.

6. Reid T, Galanis E, Abbruzzese J, et al. Intra-arterial administration of a replication-selective adenovirus (d11520) in patients with colorectal carcinoma metastatic to the liver: a phase I trial. Gene Ther. 2001;8(21): $1618-1626$.

7. Hecht JR, Bedford R, Abbruzzese JL, et al. A phase I/II trial of intratumoral endoscopic ultrasound injection of ONYX-015 with intravenous gemcitabine in unresectable pancreatic carcinoma. Clin Cancer Res. 2003;9(2):555-561.

8. Galanis E, Okuno SH, Nascimento AG, et al. Phase I-II trial of ONYX015 in combination with MAP chemotherapy in patients with advanced sarcomas. Gene Ther. 2005;12(5):437-445.

9. Reid T, Galanis E, Abbruzzese J, et al. Hepatic arterial infusion of a replication-selective oncolytic adenovirus (d11520): phase II viral, immunologic, and clinical endpoints. Cancer Res. 2002;62(21):6070-6079.

10. Khuri FR, Nemunaitis J, Ganly I, et al. a controlled trial of intratumoral ONYX-015, a selectively-replicating adenovirus, in combination with cisplatin and 5-fluorouracil in patients with recurrent head and neck cancer. Nat Med. 2000;6(8):879-885.

11. Yu W, Fang H. Clinical trials with oncolytic adenovirus in China. Curr Cancer Drug Targets. 2007;7(2):141-148.

12. Garber K. China approves world's first oncolytic virus therapy for cancer treatment. J Natl Cancer Inst. 2006;98(5):298-300.

13. Lu W, Zheng S, Li XF, Huang JJ, Zheng X, Li Z. Intra-tumor injection of H101, a recombinant adenovirus, in combination with chemotherapy in patients with advanced cancers: a pilot phase II clinical trial. World J Gastroenterol. 2004;10(24):3634-3638.

14. Xia ZJ, Chang JH, Zhang L, et al. [Phase III randomized clinical trial of intratumoral injection of E1B gene-deleted adenovirus (H101) combined with cisplatin-based chemotherapy in treating squamous cell cancer of head and neck or esophagus]. Ai Zheng. 2004;23(12):1666-1670. Chinese.

15. Kanai R, Wakimoto H, Cheema T, Rabkin SD. Oncolytic herpes simplex virus vectors and chemotherapy: are combinatorial strategies more effective for cancer? Future Oncol. 2010;6(4):619-634.

16. Geevarghese SK, Geller DA, de Haan HA, et al. Phase I/II study of oncolytic herpes simplex virus NV1020 in patients with extensively pretreated refractory colorectal cancer metastatic to the liver. Hum Gene Ther. 2010;21(9):1119-1128.

17. Fong Y, Kim T, Bhargava A, et al. A herpes oncolytic virus can be delivered via the vasculature to produce biologic changes in human colorectal cancer. Mol Ther. 2009;17(2):389-394.

18. Goins WF, Huang S, Cohen JB, Glorioso JC. Engineering HSV-1 vectors for gene therapy. Methods Mol Biol. 2014;1144:63-79. 
19. Amgen. Amgen Submits Marketing Authorization Application For Talimogene Laherparepvec To The European Medicines Agency [press release]. Thousand Oaks: Amgen; September 2, 2014. Available from: http://www.amgen.com/media/media_pr_detail. jsp?releaseID=1962767. Accessed December 4, 2014.

20. Harrington KJ, Hingorani M, Tanay MA, et al. Phase I/II study of oncolytic HSV GM-CSF in combination with radiotherapy and cisplatin in untreated stage III/IV squamous cell cancer of the head and neck. Clin Cancer Res. 2010;16(15):4005-4015.

21. Puzanov I, Milhem MM, Andtbacka RHI, et al. Primary analysis of a phase $1 \mathrm{~b}$ multicenter trial to evaluate safety and efficacy of talimogene laherparepvec (T-VEC) and ipilimumab (ipi) in previously untreated, unresected stage IIIB-IV melanoma. Presented at the 2014 ASCO Annual Meeting; May 30-June 3, 2014; Chicago, IL.

22. Clements D, Helson E, Gujar SA, Lee PWK. Reovirus in cancer therapy: an evidence-based review. Oncolytic Virother. 2014;2014(3):69-82.

23. Harrington KJ, Vile RG, Melcher A, Chester J, Pandha HS. Clinical trials with oncolytic reovirus: moving beyond phase I into combinations with standard therapeutics. Cytokine Growth Factor Rev. 2010;21(2-3): 91-98.

24. Ocean AJ, Bekaii-Saab TS, Chaudhary I, et al. A multicenter phase I study of intravenous administration of reolysin in combination with irinotecan/fluorouracil/leucovorin (FOLFIRI) in patients (pts) with oxaliplatin-refractory/intolerant KRAS-mutant metastatic colorectal cancer (mCRC). Presented at the 2013 Gastrointestinal Cancers Symposium; January 24-26, 2013; San Francisco, CA.

25. Guse K, Cerullo V, Hemminki A. Oncolytic vaccinia virus for the treatment of cancer. Expert Opin Biol Ther. 2011;11(5):595-608.

26. Vacchelli E, Aranda F, Obrist F, et al. Trial watch: Immunostimulatory cytokines in cancer therapy. Oncoimmunology. 2014;3:e29030.

27. Zhang Q, Yu YA, Wang E, et al. Eradication of solid human breast tumors in nude mice with an intravenously injected light-emitting oncolytic vaccinia virus. Cancer Res. 2007;67(20):10038-10046.
28. Heo J, Breitbach CJ, Moon A, et al. Sequential therapy with JX-594, a targeted oncolytic poxvirus, followed by sorafenib in hepatocellular carcinoma: preclinical and clinical demonstration of combination efficacy. Mol Ther. 2011;19(6):1170-1179.

29. Transgene [homepage on the Internet]. Pexa-Vec oncolytic virus: A novel targeted approach to treat solid tumors. Strasbourg: Transgene; Available from: http://www.transgene.fr/?option=com_content\&view= article\&id=82\&Itemid=76. Accessed December 4, 2014.

30. Mell LK, Yu YA, Brumund KT, et al. Phase I Trial Of Attenuated Vaccinia Virus (GL-ONC1) Delivered Intravenously With Concurrent Cisplatin And Radiotherapy In Patients With Locoregionally Advanced Head And Neck Carcinoma. Presented at the 2014 Multidisciplinary Head and Neck Cancer Symposium; February 20-22; 2014; Scottsdale, AZ.

31. Chen GX, Zhang S, He XH, Liu SY, Ma C, Zou XP. Clinical utility of recombinant adenoviral human $\mathrm{p} 53$ gene therapy: current perspectives. Onco Targets Ther. 2014;7:1901-1909.

32. Oncolytics Biotech. Oncolytics Biotech Inc. Announces Positive Top-Line Data from REO 018 Randomized Study of REOLYSIN in Head and Neck Cancers [press release]. Calgary: Oncolytics Biotech; 2013. Available from: http://www.oncolyticsbiotech.com/ news/press-release-details/2013/Oncolytics-Biotech-Inc-AnnouncesPositive-Top-Line-Data-from-REO-018-Randomized-Study-ofREOLYSIN-in-Head-and-Neck-Cancers. Accessed December 4, 2014.

33. Nowak AK, Lesterhuis WJ. Chemoimmunotherapy: still waiting for the magic to happen. Lancet Oncol. 2014;15(8):780-781.

34. Lolkema MP, Arkenau HT, Harrington K, et al. A phase I study of the combination of intravenous reovirus type 3 Dearing and gemcitabine in patients with advanced cancer. Clin Cancer Res. 2011;17(3):581-588.

35. Comins C, Spicer J, Protheroe A, et al. REO-10: a phase I study of intravenous reovirus and docetaxel in patients with advanced cancer. Clin Cancer Res. 2010;16(22):5564-5572.
Oncolytic Virotherapy

\section{Publish your work in this journal}

Oncolytic Virotherapy is an international, peer-reviewed, open access online journal publishing original research, study protocols, reviews, editorials and commentaries on all aspects of oncolytic virology, namely the application of oncolytic viruses for the treatment of cancer. Specific topics in the journal include: Rationale and theoretical aspects of oncolytic virotherapy including in vitro, in vivo and mathematical

Submit your manuscript here: http://www.dovepress.com/oncolytic-virotherapy-journal

\section{Dovepress}

modeling; and practical application and problem solving in the clinic including identification of potential responders through biomarkers and genetic profiling. The manuscript management system is completely online and includes a very quick and fair peer-review system, which is all easy to use. Visit http://www.dovepress.com/ testimonials.php to read real quotes from published authors. 